ULB-TH/10-01

\title{
The Coulomb solution as a coherent state of unphysical photons
}

\author{
Glenn Barnich ${ }^{a}$ \\ Physique Théorique et Mathématique \\ Université Libre de Bruxelles \\ and \\ International Solvay Institutes \\ Campus Plaine C.P. 231, B-1050 Bruxelles, Belgium
}

\begin{abstract}
In the context of the problem of what micro-states are responsible for the entropy of black holes, we consider as a physical toy model the electromagnetic Coulomb solution. By quantizing the electromagnetic field in the presence of an external source of charge $Q$, the quantum state corresponding to the Coulomb solution is identified as a coherent state of longitudinal and temporal photons in a Hilbert space with negative norm states.
\end{abstract}




\section{Introduction}

A major challenge in contemporary theoretical physics is a better understanding of the microscopic origin of the Bekenstein-Hawking black hole entropy [1, 2]. There have been many speculations that the degrees of freedom responsible for this entropy might have something to do with gauge degrees of freedom (see e.g. [3]) that cease to be pure gauge because of non trivial boundary conditions. This is borne out by the existence of a black hole solution in $2+1$ dimensional gravity with a negative cosmological constant [4, 5], despite the fact that there are no physical gravitons. Because of the latter feature, the problem of the entropy of the BTZ black hole is technically simpler than the one of its higher dimensional cousins, and has been analyzed extensively (see e.g. [6, 7, 8]).

From a mathematical perspective, mass and angular momentum in general relativity are akin to electric charge in pure electromagnetism: these observables are described by surface charges and are related to parameters of vanishing gauge transformations, Killing vectors of a suitable background in the former case and a constant gauge parameter in the latter [9, 10]. From this point of view, the analogue of a black hole of mass $M$ is the Coulomb solution with charge $Q$. Before turning to black holes, it is thus useful to understand the micro-states corresponding to the Coulomb solution.

The computation presented below is a straightforward exercise whose result is known in some form or the other (see e.g. section 18 of [11]) and that can easily be generalized to the coupling of the electromagnetic field with an arbitrary conserved current. Nevertheless, it seems worthwhile to provide a complete and self-contained Hamiltonian derivation, as it sheds some light on the black hole problem.

\section{Quantum Coulomb solution as a coherent state}

By Coulomb solution, we mean the solution to Maxwell's equations with zero magnetic and transverse electric fields that is produced by a static point-particle charge. For simplicity, we consider the case where the world-line of the point-particle coincides with the time-axis of Minkowski space-time. The associated action principle is given by

$$
S^{Q}=\int d^{4} x\left[-\frac{1}{4} F_{\mu \nu} F^{\mu \nu}-j^{\mu} A_{\mu}\right]
$$

with $j^{\mu}=\delta_{0}^{\mu} Q \delta^{3}(\vec{x})$.

BRST quantization of this system is well-known. We follow chapter 19 of [12] where the case $j^{\mu}=0$ has been considered in great detail. In the presence of the source,

\footnotetext{
${ }^{1}$ Note that (19.33a), (19.33d) should be multiplied by $\sqrt{2}$ and (19.33b), (19.33c) divided by $\sqrt{2}$ in order to yield the BRST charge (19.34).
} 
the secondary constraint, Gauss's law, becomes $\phi_{2}^{Q} \equiv-\pi^{i}{ }_{i}+j^{0}=0$ and the BRST charge is modified to

$$
\Omega^{Q}=\int d^{3} k c^{*}(\vec{k})[a(\vec{k})-q(\vec{k})]+\left[a^{*}(\vec{k})-q(\vec{k})\right] c(\vec{k}),
$$

where $a(\vec{k})=a_{3}(\vec{k})+a_{0}(\vec{k})$, with $a_{3}(\vec{k}), a_{0}(\vec{k})$ the oscillators associated with longitudinal and temporal photons respectively and $q(\vec{k})=\frac{Q}{(2 \pi)^{3 / 2} \sqrt{2} k^{3 / 2}}$.

The BRST operator $\widehat{\Omega}^{Q}$ does not any longer annihilate the standard vacuum. In order to have the usual quartet mechanism [13] at work, one can define the shifted oscillators $a^{Q}(\vec{k})=a(\vec{k})-q(\vec{k}), a^{* Q}=a^{*}(\vec{k})-q(\vec{k})$ without affecting the commutation relations and define the appropriate vacuum through

$$
\widehat{a}^{Q}(\vec{k})|0\rangle^{Q}=0
$$

with $|0\rangle^{Q}$ being annihilated by all the other destruction operators. In terms of the standard vacuum, the new vacuum appears as a coherent state made out of temporal and longitudinal photons,

$$
|0\rangle^{Q}=\prod_{\vec{k}} \exp q(\vec{k}) \widehat{b}^{\dagger}(\vec{k})|0\rangle=\exp \int d^{3} k q(\vec{k}) \widehat{b}^{\dagger}(\vec{k})|0\rangle,
$$

with $b(\vec{k})=\frac{1}{2}\left(a_{3}(\vec{k})-a_{0}(\vec{k})\right)$ and $\left[\widehat{a}(\vec{k}), \widehat{b}^{\dagger}\left(\vec{k}^{\prime}\right)\right]=\delta^{3}\left(\vec{k}-\vec{k}^{\prime}\right)$. Because $\widehat{b}^{\dagger}(\vec{k})$ are null oscillators, ${ }^{Q}\langle 0 \mid 0\rangle^{Q}=\langle 0 \mid 0\rangle=1$. As we are now going to show, it is the state $|0\rangle^{Q}$ that corresponds to the Coulomb solution.

Indeed, the expectation value of $\widehat{A}_{\mu}(\vec{x})$ in the new vacuum is

$$
{ }^{Q}\left\langle 0\left|\widehat{A}_{\mu}(\vec{x})\right| 0\right\rangle^{Q}=\delta_{\mu}^{0} \frac{1}{(2 \pi)^{3 / 2}} \int d^{3} k \frac{Q}{(2 \pi)^{3 / 2} 2 k^{2}} \exp i \vec{k} \vec{x}=\delta_{\mu}^{0} \frac{Q}{8 \pi r} .
$$

With $A_{i}=0$, the classical equations of motion are solved by

$$
A_{\mu}(\vec{x})=\delta_{\mu}^{0} \frac{Q}{4 \pi r},
$$

which differs from (2.5) by a factor 2 . There is however no contradiction as

$$
{ }^{Q}\left\langle 0\left|\partial_{i} \widehat{A}^{i}(x)\right| 0\right\rangle^{Q} \neq 0 \text {. }
$$

even though ${ }^{Q}\left\langle 0\left|\widehat{A}_{i}(x)\right| 0\right\rangle^{Q}=0$ and thus $\partial_{i}{ }^{Q}\left\langle 0\left|\widehat{A}^{i}(x)\right| 0\right\rangle^{Q}=0$ since the polarization vector $e_{i}^{3}(\vec{k})$ is odd under $\vec{k} \rightarrow-\vec{k}$, while $k^{i} e_{i}^{3}(\vec{k})$ is even.

The expectation values of the gauge invariant electric and magnetic fields in the new vacuum agree with their classical analogs:

$$
\begin{aligned}
{ }^{Q}\left\langle 0\left|\widehat{\pi}^{i}(x)\right| 0\right\rangle^{Q} & ={ }^{Q}\left\langle 0\left|-\frac{i}{(2 \pi)^{3 / 2}} \int d^{3} k \sqrt{\frac{k}{2}}\left[a e_{3}^{i} \exp i \vec{k} \vec{x}-a^{*} e_{3}^{i} \exp -i \vec{k} \vec{x}\right]\right| 0\right\rangle^{Q} \\
& =-\frac{2 i}{(2 \pi)^{3 / 2}} \int d^{3} k \sqrt{\frac{k}{2}} q(\vec{k}) \frac{k_{i}}{k} \exp i \vec{k} \vec{x} \\
& =-\frac{Q}{(2 \pi)^{3}} \frac{\partial}{\partial x^{i}} \int d^{3} k \frac{1}{k^{2}} \exp i \vec{k} \vec{x}=\frac{Q x^{i}}{4 \pi r^{3}}
\end{aligned}
$$


while

$$
{ }^{Q}\langle 0|\vec{\nabla} \times \widehat{\vec{A}}| 0\rangle^{Q}=0
$$

because $\epsilon^{i j l} k_{j} e_{l}^{3}(\vec{k})=0$.

\section{Discussion}

Since it is built out of longitudinal and temporal photons, understanding the Coulomb solution quantum mechanically is thus only possible if one quantizes all polarizations in a Hilbert space with negative norm states and not if one reduces to physical degrees of freedom around the new vacuum before quantization. The advantage as compared to the $A_{0}=0$ gauge advocated for instance in [14] is that a charge eigenstate now appears as a displaced vacuum state. This generalizes to unphysical photons the corresponding property of physical photons when coupled to a classical external vector current density [15].

\section{Acknowledgments}

The author wants to thank M. Bañados, J. Evslin, A. Gomberoff, C. Krishnan, C. Maccaferri, C. Schomblond and S. Theisen for useful discussions. This work is supported in part by the Fund for Scientific Research-FNRS (Belgium), by the Belgian Federal Science Policy Office through the Interuniversity Attraction Pole P6/11, by IISN-Belgium, by Fondecyt projects No. 1085322 and No. 1090753 and by the Perimeter Institute for Theoretical Physics.

\section{References}

[1] J. D. Bekenstein, "Black holes and entropy,” Phys. Rev. D7 (1973) 2333-2346.

[2] S. W. Hawking, "Particle creation by black holes," Commun. Math. Phys. 43 (1975) 199-220.

[3] G. 't Hooft, "Space-time and quantum mechanics," in 23rd International Solvay Conference in Physics: The Quantum Structure of Space and Time. 2005.

[4] M. Bañados, C. Teitelboim, and J. Zanelli, "The black hole in three-dimensional space-time," Phys. Rev. Lett. 69 (1992) 1849-1851, hep-th/9204099. 
[5] M. Bañados, M. Henneaux, C. Teitelboim, and J. Zanelli, "Geometry of the (2+1) black hole," Phys. Rev. D48 (1993) 1506-1525, gr-qc/9302012.

[6] S. Carlip, "The statistical mechanics of the (2+1)-dimensional black hole," Phys. Rev. D51 (1995) 632-637, gr-qc/9409052.

[7] S. Carlip, "What we don't know about BTZ black hole entropy," Class. Quant. Grav. 15 (1998) 3609-3625, hep-th/9806026.

[8] A. Strominger, "Black hole entropy from near-horizon microstates," JHEP 02 (1998) 009, arXiv: hep-th/9712251

[9] L. F. Abbott and S. Deser, "Stability of gravity with a cosmological constant," Nucl. Phys. B195 (1982) 76.

[10] L. F. Abbott and S. Deser, "Charge definition in nonabelian gauge theories," Phys. Lett. B116 (1982) 259.

[11] N. Papanicolaou, "Infrared problems in quantum electrodynamics," Phys. Rept. 24 (1976) 229-313.

[12] M. Henneaux and C. Teitelboim, Quantization of Gauge Systems. Princeton University Press, 1992.

[13] T. Kugo and I. Ojima, "Local covariant operator formalism of nonabelian gauge theories and quark confinement problem," Prog. Theor. Phys. Suppl. 66 (1979) 1.

[14] J.-L. Gervais and B. Sakita, "Gauge degrees of freedom, external charges, and quark confinement criterion in the a $(0)=0$ canonical formalism," Phys. Rev. D18 (1978) 453.

[15] R. J. Glauber, "Coherent and incoherent states of the radiation field," Phys. Rev. 131 (1963) 2766-2788. 